\title{
Implementación de una innovación tecnológica. Espacio de formación multimodal, e-UAEM
}

\author{
Implementation of a technological innovation. Multimodal training \\ space, e-UAEM
}

\section{Maribel Castillo Díaz María Luisa Zorrilla Abascal}

Universidad Autónoma del Estado de México (UAEM), México

\section{Resumen}

Este trabajo es parte de los resultados de la tesis "Análisis del proceso de implementación de un entorno virtual de aprendizaje en educación superior: estudio de caso e-UAEM (Espacio de Formación Multimodal)", realizada en la Universidad Autónoma del Estado de Morelos (UAEM) México.

El objetivo general de la investigación fue caracterizar, analizar y comprender, el proceso de implementación de una solución educativa de educación superior basada en un entorno virtual de aprendizaje, en este caso particular, e-UAEM.

Uno de los objetivos específicos fue "identificar y describir la concepción del Espacio de Formación Multimodal de la Universidad Autónoma del Estado de Morelos" con el fin de conocer la percepción de los actores respecto del Espacio de Formación Multimodal en la UAEM. Fue un estudio cualitativo en el cual se entrevistaron docentes, directivos e implementadores del proyecto; se realizó análisis documental y grupo focal con las personas involucradas desde la dimensión académica de e-UAEM. La perspectiva teórica fue sobre el cambio organizacional.

Palabras clave: formación multimodal; innovación tecnológica; cambio organizacional.

\begin{abstract}
This work is part of the results of the thesis "Analysis of the implementation process of a virtual learning environment in higher education: case study e-UAEM (Multimodal Education Space)", held in UAEM, the acronym for Universidad Autónoma del Estado de Morelos, México.

The general objective of the research was to characterize, analyze and understand the process of implementing an educational solution of higher education based on a virtual learning environment, in this particular case, e-UAEM.

One of the specific objectives was to "identify and describe the conception of the Multimodal Education Space of the Universidad Autónoma del Estado de Morelos" in order to know the perception of the actors regarding the Multimodal Education Space in the UAEM. It was a qualitative study in which teachers, managers and implementers of the project were interviewed; documentary analysis and focus group were carried out with the people involved from the academic dimension of e-UAEM. The theoretical perspective was about organizational change.
\end{abstract}

Keywords: Multimodal Education; technological innovation; organizational change. 


\section{INTRODUCCIÓN}

En América Latina desde hace más de dos décadas la cobertura es insuficiente en el nivel Superior. El porcentaje de individuos, entre los 18 y 24 años de edad, inscritos en educación superior en ALC creció de 21 por ciento en 2000 a 40 por ciento en 2010 (Banco mundial, 2017), sin embargo, aún no se desarrolla en todo su potencial. En el caso de México según datos del Informe Nacional sobre la Educación Superior (2003: 50 y 51), se distribuye de manera desigual por entidades. Por su parte el Centro de Estudios Sociales y de Opinión Pública CESOP (2005) vislumbró que, en 2005, ante los resultados de los cambios demográficos en México durante el siglo $X X I$, las instituciones de educación superior presentarían un aumento significativo de demanda estudiantil en los siguientes años. Se anticipó que en 2013 se alcanzaría un máximo de 14.9 millones de personas en edad potencial de cursar educación superior, de manera que entre 2000 y 2013 la demanda se incrementaría un 6.9 $\%$, situación que en la UAEM se ha visto confirmada. Ante esta situación en esta institución, las primeras iniciativas para atender esta problemática con el uso de las TIC iniciaron en 2009 cuando se incorpora el proyecto de formación multimodal, cuyo principal objetivo fue complementar los espacios físicos de la institución con espacios virtuales. Esta propuesta abre las posibilidades de cobertura desde una formación multimodal que abarca desde la oferta educativa 100\% presencial, hasta la 100\% en línea, pasando por una variedad de combinaciones intermedias denominadas "híbridas". (UAEM-Gaceta, 2007).

La ampliación de la cobertura es uno de los fines principales que se persiguen al hibridar o virtualizar programas educativos en la UAEM, desde dicho fin se desprenden tres objetivos que e-UAEM se planteó desde su surgimiento: a) Flexibilizar y enriquecer la oferta presencial; b) Incorporar el uso de las TIC a los procesos de enseñanza-aprendizaje; y c) Ampliar el acceso, es decir, hacer más inclusiva la educación (Zorrilla, 2009a).

Lo anteriormente expresado es coincidente con el Programa Institucional de Desarrollo Educativo 2007-2013 de la UAEM que enuncia:

La ampliación de la cobertura debe sustentarse no sólo en el uso de tecnologías que permiten la educación a distancia, sino en modalidades de educación presencial creativas, en donde sea la universidad la que vaya al alumno, y no el alumno a las instalaciones de la universidad, a través de modalidades flexibles de enseñanza, de la integración de programas y del uso compartido de recursos. (UAEM, 2007: 23). 
La introducción de la formación multimodal en la Universidad implicó un cambio de cultura organizacional en varias esferas de la institución, sin embargo, para este estudio sólo se hará referencia a las implicaciones que conllevan la compresión del concepto de formación multimodal por parte de los actores de las áreas de gestión involucrados.

\section{PERSPECTIVA METOdOLÓGICA}

De acuerdo a las preguntas de investigación y objetivos y después de haber revisado la literatura relacionada con el tema de TIC y educación superior, se optó por un abordaje metodológico de corte cualitativo. La aproximación cualitativa permitió obtener una visión integral del proceso de implementación de e-UAEM a partir de tres perspectivas: 1) Gestión: centrada en la dimensión político-administrativa del proceso; 2) Académica: centrada en la formación docente, la producción y el uso de contenidos educativos; 3) Tecnológica: centrado en la infraestructura y los servicios tecnológicos. En este estudio sólo se aborda parte de la primera dimensión, en específico la conceptualización de la formación multimodal.

Fue un estudio de caso y se apoyó de las siguientes técnicas: análisis de documentos, e-observación semi-estructurada, entrevista semiestructurada y grupo focal. Los audios de las entrevistas y del grupo focal se transcribieron con F4, un programa gratuito que permite sincronizar los datos con el programa de análisis cualitativo Atlas.ti.

\section{PERSPECTIVA TEÓRICA}

A partir de la revisión de diversos referentes teóricos y del análisis de los datos recabados en esta investigación, se organizaron los resultados a partir de las propuestas teóricas del cambio organizacional. Esta decisión se basa en la comprensión de la implementación de una innovación educativa, en este caso el Espacio de Formación Multimodal (e-UAEM), como un proceso complejo y multidimensional que impacta en la estructura, las prácticas académicas y administrativas, la cultura organizacional y los recursos de la institución, constituyendo, por tanto, ejes de un potencial cambio organizacional. 
Cabe mencionar que se considera como una innovación al tratarse de una propuesta en donde a través de la oferta de asignaturas híbridas y virtuales de programas educativos combinan diferentes modalidades y plantea un escenario de flexibilidad modal con el fin de aprovechar las ventajas que cada una de ellas representa, permitiendo así, ampliar la cobertura en el nivel superior. Esta propuesta nos hace retomar el concepto de cambio, ya que ante la integración de la formación multimodal se requirió de transformación en varias esferas del ámbito académico (formación docente, estrategias didácticas), organizativo (procesos administrativos, financieros), y tecnológico (capacitación).

De acuerdo con diferentes autores, el cambio es un proceso de transición concertado y planificado que implica que una organización pase de su estado actual a otro que se desea en el futuro; a fin de que la institución sea más eficiente y mejore la calidad de sus servicios, mediante el uso racional de sus recursos, la mejora de sus relaciones inter e intra institucionales y el desarrollo de sus propias capacidades (conocimientos y habilidades) (Jones, 2008; Cummings y Worley, 2005).

No obstante, si bien el cambio organizacional es una noción que nos ayuda a comprender el proceso de implementación de e-UAEM, esta investigación arroja claramente que, contrario a la ortodoxia planteada por los autores antes mencionados, el cambio puede darse de formas menos estructuradas, como ha sucedido en el caso que nos ocupa. Esto lo expresa Acosta (2002: 22) en los siguientes términos: el cambio organizacional "consiste en el conjunto de transformaciones que se realizan en las distintas dimensiones de las organizaciones, es producido tanto por fuerzas naturales como impulsado por la voluntad de quienes las crean y las impulsan". Asimismo, plantea cuatro preguntas para comprender el cambio organizacional:

- ¿Qué es el cambio organizacional?

- ¿Por qué cambian las organizaciones?

- ¿Qué es lo que cambia en las organizaciones?

- ¿Cómo cambian las organizaciones?

Estas preguntas operan como categorías de análisis para la revisión de los resultados derivados de esta investigación, permitiéndonos comprender y dar cuenta del cambio que representa la implementación de un entorno virtual de enseñanza y aprendizaje dentro de una institución de educación superior. 
De las preguntas antes planteadas, consideramos que la forma más visible del cambio es identificar qué es lo que se transforma en la organización.

Ante la interrogante de ¿Qué es lo que cambia en las organizaciones?, el autor mencionado responde que «...el cambio organizacional ocurre en la totalidad de la organización o en sus estructuras, en los procesos, las áreas o las dimensiones (política, de funcionamiento interno, de relaciones exteriores) (2002:10). El mismo autor revisa diferentes aproximaciones teóricas que proponen respuestas diversas a la pregunta de referencia:

- Gordon (1999: 675) plantea que los cambios se producen en los sistemas técnicos, sociales, administrativos o estratégicos.

- Leavitt (citado por Bartlett 1987) entiende el cambio como un proceso que incide en una o varias de las siguientes variables: la tarea, la estructura, la tecnología y/o los actores.

- Schvarstein (1992) afirma que el cambio organizacional se da en los significados y, por tanto, en la cultura.

Identificar los aspectos en los que se manifiesta el cambio organizacional funciona en este apartado como organizador temático para estructurar los resultados según los aspectos principales que detectamos como transformados o en proceso de transformación a partir de la implementación de e-UAEM. En cada uno de estos aspectos, exploramos el qué, el por qué y el cómo del cambio.

Conforme a los datos obtenidos y analizados, los seis temas más relevantes en los que se manifiesta el cambio organizacional a partir de la implementación de e-UAEM son los siguientes:

- Innovación pedagógica partir del concepto de multimodalidad

- Políticas y tendencias de las TIC en educación superior

- Estructura organizacional

- Gestión del cambio en los procesos organizacionales

- Cultura organizacional

- Infraestructura tecnológica

En este documento sólo se abarca a la primera temática "Innovación pedagógica a partir del concepto de multimodalidad" que a continuación se presenta. 


\section{INNOVACIÓN A PARTIR DEL CONCEPTO DE MULTIMODAL}

\subsection{Antecedentes TIC en la UAEM}

Las iniciativas para integrar las Tecnologías de Información y Comunicación (TIC) en la Universidad Autónoma del Estado de Morelos en el nivel de Educación Superior iniciaron en 2005, cuando por primera vez se ofertó el programa de Contador Público de la Facultad de Contaduría, Administración e Informática (FCAel), a través del Sistema de Educación Abierta y a Distancia (SEAD) y con la implementación en modalidad no-convencional de un programa de posgrado de la Facultad de Psicología.

En la tabla 1 se observa la cronología de la integración de las TIC antes de la creación de e-UAEM en esta universidad. Como se puede apreciar, en septiembre de 2008 no había evidencia de la existencia de un proyecto o programa institucional para incorporar las TIC. Sino que fue hasta octubre de 2009 cuando las primeras iniciativas de implementación de las TIC en la UAEM se unificaron en un solo proyecto institucional, denominado Espacio de Formación Multimodal (e-UAEM), cuya finalidad se planteó en términos de ampliar la cobertura educativa del nivel superior complementando los espacios físicos con espacios virtuales; y generar una solución transversal institucional para la adquisición y desarrollo de competencias TIC por parte de estudiantes y docentes. La característica principal de esta propuesta fue la multimodalidad, que abarca desde la oferta educativa 100\% presencial, hasta la 100\% en línea, pasando por una variedad de combinaciones intermedias denominadas "híbridas" (UAEM-Gaceta, 2012).

\section{TABLA 1}

Cronología de integración de modalidades educativas no-convencionales en la UAEM (1978-2008)

\begin{tabular}{|c|c|}
\hline Año & $\begin{array}{c}\text { Iniciativa o proyecto relacionado con modalidades educativas } \\
\text { no-convencionales en la UAEM }\end{array}$ \\
\hline 1978 & Se crea el Sistema de Enseñanza Abierta para Bachillerato \\
\hline 1998 & $\begin{array}{l}\text { Se aprueba el Plan de Estudios de Bachillerato del Sistema de Educación Abierta y } \\
\text { a Distancia (SEAD) }\end{array}$ \\
\hline \multirow[t]{3}{*}{2005} & $\begin{array}{l}\text { Se aprueban los planes de estudios de las licenciaturas de Contador Público y } \\
\text { Administración en modalidad no convencional y a distancia de la Facultad de Contaduría, } \\
\text { Administración e Informática (FCAel). }\end{array}$ \\
\hline & $\begin{array}{l}\text { Inicia la licenciatura de Contador Público de la FCAel a través del Sistema de Educación } \\
\text { Abierta y a Distancia (SEAD). }\end{array}$ \\
\hline & $\begin{array}{l}\text { Se implementa la Especialidad en Problemas de Farmacodependencia de la Facultad } \\
\text { de Psicología (modalidad no-convencional), la cual hasta la fecha ha tenido una sola } \\
\text { generación. }\end{array}$ \\
\hline
\end{tabular}




\begin{tabular}{|l|l|}
\hline 2006 & $\begin{array}{l}\text { Inicia la licenciatura en Administración de la Facultad de Contaduría, } \\
\text { Administración e Informática a través del Sistema de Educación Abierta y a } \\
\text { Distancia (SEAD). }\end{array}$ \\
& $\begin{array}{l}\text { Cursos de formación docente relacionadas con el uso de las TIC organizados por } \\
\text { el departamento de Actualización y Capacitación Docente, de la Dirección de } \\
\text { Educación Superior, UAEM: } \\
\text { - La docencia con el uso de las TICs (31/05/2006- 02/06/2006) }\end{array}$ \\
\hline 2007 & $\begin{array}{l}\text { Cursos de formación docente relacionadas con el uso de las TIC organizados por el } \\
\text { departamento de Actualización y Capacitación Docente, de la Dirección de Educación } \\
\text { Superior, UAEM: } \\
\text { - Aplicación de las tecnologías de la información y la comunicación en la tarea docente } \\
\text { (5/31/2007- 02/06/2007) } \\
\text { - El papel de las TICs en la formación del asesor universitario (16/07/2007-19/07/2009) } \\
\text { - Aplicación de las tecnologías de la información y la comunicación en el desarrollo del } \\
\text { docente universitario (03/09/2007-07/09/2007) }\end{array}$ \\
\hline $\begin{array}{l}\text { Maestría en Nutrición, Salud y Dietética, de la Facultad de Medicina (en modalidad mixta) } \\
\text { Decisión de la Rectoría 2007- 2012 de abrir programas de educación a distancia para } \\
\text { ampliar la cobertura en educación superior. }\end{array}$ \\
$\begin{array}{l}\text { Cursos de formación docente relacionadas con el uso de las TIC organizados por } \\
\text { el departamento de Actualización y Capacitación Docente, de la Dirección de } \\
\text { Educación Superior, UAEM: }\end{array}$ \\
$\begin{array}{l}\text { - El uso de las TICs en la formación del profesor universitario (28/01/2008-01/02/2008) } \\
\text { - El papel delas TICsen la formación del profesorado universitario (18/08/2008-22/08/2008) } \\
\text { - Desarrollo de material multimedia (22/09/2008-26/09/2008) } \\
\text { - Diplomado: Formación docente en ambientes virtuales. Ofertado por la Universidad } \\
\text { Oberta de Catalunya. }\end{array}$ \\
\hline
\end{tabular}

Fuente: (Castillo, 2014: 89).

\subsection{Concepciones de modalidades}

Introducir una nueva forma de nombrar algo que conocíamos anteriormente con otro nombre no es fácil. Más complejo aún es introducir una palabra con la que no estábamos familiarizados y cuyo significado es complejo. Estos cambios implican la deconstrucción y reconstrucción de estructuras conceptuales que forman parte de las culturas organizacionales, de los imaginarios y representaciones de una comunidad.

La implementación de e-UAEM, en un principio, representó precisamente la irrupción de un nuevo vocablo en la vida universitaria: multimodal. Pero no sólo era una nueva palabra, o una palabra de moda, sino que entrañaba una nueva forma de pensar las modalidades educativas. En este sentido, situamos la concepción de lo multimodal como una innovación que fue el principio de la oportunidad de cambio organizacional. 
Para situar al Espacio de Formación Multimodal como una innovación, es necesario primero abordar las concepciones de las diferentes modalidades educativas. La Ley General de Educación' (2006: 13) en el artículo 46 refiere: "La educación tendrá las modalidades de escolar, no escolarizada y mixta", modalidades que se fusionan e interoperan e-UAEM. En este sentido Zorrilla y Castillo (2013) resumen las modalidades de la siguiente forma:

\section{TABLA 2}

\section{Resumen de las tres modalidades reconocidas por la SEP}

\begin{tabular}{|c|c|c|}
\hline $\begin{array}{c}\text { Modalidad } \\
\text { (Ley General } \\
\text { de Educación) }\end{array}$ & Nombres alternativos & Principales características \\
\hline Escolar & $\begin{array}{l}\text { - Escolarizada } \\
\text { - Presencial } \\
\text { - Tradicional } \\
\text { - Convencional }\end{array}$ & $\begin{array}{l}\text { - Docente y estudiantes coinciden en tiempo } \\
\text { y espacio. } \\
\text { - El espacio educativo es físico y formal: aula, } \\
\text { laboratorio y auditorio. Generalmente dentro } \\
\text { de una institución educativa. }\end{array}$ \\
\hline No escolarizada & $\begin{array}{l}\text { - A distancia } \\
\text { - Educación virtual } \\
\text { - e-learning (electronic } \\
\text { learning) } \\
\text { - Educación en línea } \\
\text { - No-convencional }\end{array}$ & $\begin{array}{l}\text { - Docente y estudiantes se encuentran espa- } \\
\text { cialmente separados (a distancia) } \\
\text { - El espacio educativo es virtual y puede ser } \\
\text { formal (plataforma educativa, software de te- } \\
\text { leconferencia) o informal (blog, redes sociales). } \\
\text { - Pueden o no confluir en el tiempo (sincrónica } \\
\text { o asincrónica) } \\
\text { - Basada en TIC } \\
\text { - Uso predominante de internet }\end{array}$ \\
\hline Mixta & $\begin{array}{l}\text { - Semiescolarizada } \\
\text { - Semipresencial } \\
\text { - } \text { b-learning (blended } \\
\text { learning) } \\
\text { - Híbrida } \\
\text { - Abierta } \\
\text { - No-convencional }\end{array}$ & — Combina modalidades presencial y virtual. \\
\hline
\end{tabular}

Fuente: Zorrilla y Castillo (2013).

Las llamadas 'modalidades no-convencionales' generalmente son las que se ubican en los ámbitos de lo no-escolarizado o lo mixto. En este sentido, las instituciones de educación superior en el mundo han creado proyectos o programas para integrar

\footnotetext{
${ }^{1}$ Señala las normas generales para regular el Servicio Público de la Educación en México, que cumple una función social acorde con las necesidades e intereses de las personas, de la familia y de la sociedad. Se fundamenta en los principios de la Constitución Política sobre el derecho a la educación que tiene toda persona, en las libertades de enseñanza, aprendizaje, investigación y cátedra y en su carácter de servicio público (OIE,1994).
} 
las TIC, y se han nombrado de diferentes formas, abarcando conceptos tales como educación virtual, e-learning, b-learning, educación a distancia en línea, modalidad híbrida o mixta, y, por supuesto, formación multimodal, que es la denominación de nuestro objeto de estudio.

De acuerdo a García Aretio (2007: 2), desde una perspectiva no restringida, todas las formulaciones sobre aprendizaje electrónico, teleaprendizaje, e-learning y aprendizaje virtual, se pueden integrar dentro de la denominación matriz de educación a distancia, definida como "diálogo didáctico mediado entre el equipo docente y el estudiante que, ubicado en espacio diferente al de aquél, aprende de forma flexible, independiente y colaborativa".

La educación virtual o e-learning es una forma de enseñanza a distancia con uso predominante del Internet como medio tecnológico (García Aretio, 2002). El término de educación virtual corresponde sobre todo a la terminología que emplean las universidades, siendo e-learning un término que se ha adoptado de manera predominante en el campo de la capacitación empresarial.

Otro de los conceptos importantes a tomar en cuenta para la comprensión del concepto de formación multimodal es el de blended learning o b-learning. Bartolomé (2004) puntualiza que para abordar este concepto es necesario primero referir al fracaso del e-learning, sosteniendo que éste no ha respondido a las expectativas que había creado, por lo que ha dado paso a que se contemplen otras modalidades alternativas como el $B$-learning, para responder a las necesidades de una educación de calidad en el contexto de la educación superior.

$B$-Learning es la abreviatura de blended learning es un término inglés que se traduce como "Aprendizaje Mezclado", "Formación Combinada" o "Enseñanza Mixta". Se trata de una modalidad educativa semipresencial que incluye tanto formación no presencial (cursos on-line, conocidos genéricamente como e-learning), como formación presencial. Turpo (2010) lo define como una modalidad que representa un gran cambio en las estrategias de enseñar y aprender. Según este autor, se trata de una nueva modalidad que ha recibido varios nombres como modalidad combinada, mixta, bimodal, semipresencial, dual, híbrida o integrada, (re)conocida más ampliamente como blended learning.

De la anterior concepción se difiere en cuanto a que es una modalidad "nueva", considerando que, de acuerdo a Garrison (2007), el aprendizaje mixto no es nuevo. La novedad que introduce es el reconocimiento de su potencial para rediseñar radicalmente la experiencia de enseñanza y aprendizaje en la educación superior. 
Por su parte, Michael Fox (2002, citado por Mortera, 2009: 130-131) recupera los componentes esenciales del aprendizaje combinado y lo define como "la habilidad de combinar elementos de capacitación e instrumentación en el salón de clase, de aprendizaje a distancia en vivo y autónomo, y de servicios de aprendizaje avanzados que dan soporte de manera tal que proveen de un aprendizaje a la medida del usuario".

A lo largo de la revisión de las conceptualizaciones del blended learning se encontró que, en el ámbito educativo, se le nombra como aprendizaje mezclado, mixto (desde la perspectiva de la SEP) e híbrido. Es importante señalar que, aunque la mayoría de los autores definen este concepto a partir de la combinación de lo presencial con lo virtual, no se encuentra una mayor precisión respecto a las proporciones de dicha combinación, aunque en general podría inferirse que se trata de un 50-50\%. Tampoco se define con claridad cómo es que se combinan dichas modalidades en el tiempo y el espacio, por lo que un desafío conceptual importante es, a partir de las prácticas que se han establecido a este respecto, definir con mayor precisión cómo se mezclan estas modalidades en su diseño didáctico e implementación.

Además de las modalidades no-convencionales, para comprender Espacio de Formación Multimodal es necesario explorar la concepción del 'espacio', en tanto representación de los espacios del mundo físico, así como lo 'multimodal'. En este sentido, respecto a su concepción esencial, y no funcional, Zorrilla (2012: 17) refiere que "el Espacio de Formación Multimodal en la Universidad Autónoma del Estado de Morelos, como su nombre lo indica, es un espacio complementario que extiende las instalaciones de la UAEM más allá del ámbito físico". En tal sentido, refiriendo a la transformación del "espacio", a la fecha cuenta con aulas, laboratorios, cubículos de tutoría individual, cubículos de tutoría grupal, salas de profesores, salas de estudiantes y espacios colaborativos en línea.

Siguiendo las ideas de Zorrilla (2009b), con relación al concepto 'multimodal', podemos derivar que se refiere a la interpenetración de elementos, a la permeabilidad entre las distintas modalidades. Esto lo ejemplifica destacando que en las instituciones de educación superior es común encontrar cursos presenciales que cuentan con componentes en línea (apuntes, blogs, podcasting y foros de discusión), así como cursos a distancia que buscan ofrecer una experiencia lo más cercana posible a la vivencia presencial (discusiones sincrónicas en línea con apoyo de webcams). 
Fernández (2011) refiere que la comunicación ocurre a través de diferentes modos de significación (texto, imágenes, gráficos, sonido, música, etc.) de manera simultánea. Desde este punto de vista, el concepto multimodal aborda las características de cada modo, su interrelación y su valoración, dependiendo de la cultura en donde se enmarcan.

La multimodalidad, por ser un término centrado en el sujeto que aprende, refiere también a una competencia que, según Kress (2003) es una característica de los modos de alfabetización modernos, que demanda la habilidad para leer y expresar ideas a través de un amplio rango de modos de representación y significación.

El Espacio de Formación Multimodal de la UAEM constituye una propuesta educativa que combina formación presencial y virtual, es decir, diversos modos de aprender, en diferentes proporciones. Se trata de una multiplicidad de combinatorias posibles que, en gran medida, dependen de las necesidades y circunstancias particulares de cada estudiante, llegando incluso a altos porcentajes o a la totalidad del aprendizaje en línea, para quienes la formación presencial es en extremo improbable (Zorrilla, 2009a: 4).

Otro de los conceptos a tomar en cuenta para poder redondear la definición del "Espacio de Formación Multimodal", tiene que ver con la "formación multimodal". En el proceso de revisión de la literatura en el ámbito nacional sólo se encontró el proyecto piloto de "sistema multimodal" de la Universidad Veracruzana, que se acerca a la conceptualización del término en e-UAEM, al contemplar el b-learning como estrategia y al estar centrado en el aprendizaje y no en la enseñanza. Sin embargo, la noción de multimodalidad difiere porque se inscribe en una propuesta de "sistema", como algo diferenciado a las otras modalidades. Es decir, como una alternativa modal distinta a las demás que ofrece la universidad. En términos del discurso institucional, se define el "sistema multimodal" de la Universidad Veracruzana como "una propuesta educativa integral y de calidad fundada en las necesidades y características del aprendiente, procurando el respeto a los estilos y preferencias hacia el aprendizaje, así como a la diversidad cultural de su comunidad académica" (García y Calderón 2010: 7).

A continuación, se analizan las diferentes concepciones respecto de la formación multimodal por parte de algunos de los involucrados en el proceso de implementación de e-UAEM, pretendiendo de esta manera, obtener una diversidad de miradas que 
nos permite identificar que, desde su inicio, uno de los desafíos del proyecto fue la (in)comprensión del concepto de formación multimodal, a menudo confundido con las modalidades híbrida o virtual.

\section{DIVERSAS CONCEPCIONES DEL ESPACIO DE FORMACIÓN MULTIMODAL2}

\subsection{Docentes}

A efectos de esta investigación y considerando que los docentes son concebidos en el proceso de implementación de e-UAEM como agentes de cambio, se determinó necesario conocer cómo entendían éstos el concepto del Espacio de la Formación Multimodal que se estaba introduciendo en la UAEM. Para tal fin se inició un trabajo de indagación acerca de la percepción y/o conceptualización de la "formación multimodal".

En tal sentido, se utilizó información del curso de Asesoría y Producción de Recursos en Línea $(A P C L)^{3}$, específicamente de la actividad Mapa conceptual de formación multimodal y sus características.

De un total de 48 docentes en el curso, 39 participaron en esta actividad. Se deconstruyeron los mapas conceptuales generados por los profesores participantes yse analizó y categorizó la información recabada mediante el uso del software Atlas-ti.

La definición más generalizada entre los participantes (8 en total) se ejemplifica a partir de lo referido por el docente 9: "La formación multimodal combina procesos de enseñanza y aprendizaje en aulas presenciales y virtuales".

Por su parte, el docente 22 abunda en torno a la percepción del concepto:

"La combinación de procesos de enseñanza-aprendizaje presenciales y virtuales, que incluyen: formas híbridas o mixtas de aprendizaje. Propicia una formación centrada en el estudiante, quien desarrolla competencias relacionadas con el uso de las TIC. El alumno trabaja a su propio ritmo y organiza sus tiempos de estudio..."

\footnotetext{
2 El análisis de estos resultados a detalle se encuentran el artículo: Zorrilla, García y Castillo (2013). Formación docente en línea a partir de una estrategia de producción de contenidos para ambientes virtuales de aprendizaje.

${ }^{3}$ El registro de la e-observación se hizo en 2011 a través de una guía de ítems, que se desprendieron de las preguntas generales de investigación.
} 
Otros profesores definieron la formación multimodal como:

- Combinación de modalidades

- Cursos de educación: presenciales, semi-presenciales y a distancia

- Combinación de contenidos presenciales y virtuales

- Diferentes alternativas de aprendizaje

- Entornos virtuales: no convencional, a distancia y en línea

- Modalidades escolarizado, semiescolarizada, abierta y a distancia

- Proceso de innovación pedagógica

Doce profesores sólo refirieron a beneficios, características u origen de e-UAEM, en su carácter de espacio de Formación Multimodal sin definir el concepto de formación multimodal. Es posible que para estos profesores la actividad resultara complicada, ya que por una parte tenían que definir el concepto de formación multimodal y por otra tenían que desarrollar un mapa conceptual mediante la utilización de una herramienta tecnológica (CmapTools, PowerPoint u otro programa). Esto implicaba un triple reto, ya que no sólo demandó definir el concepto, sino que también en algunos casos los docentes aprendieron a elaborar un mapa conceptual y/o a utilizar alguna aplicación digital.

Del total de docentes que realizaron con éxito esta actividad en función de la demanda, el 33\% se inclina por definir la formación multimodal como una combinación de procesos de enseñanza-aprendizaje presenciales y virtuales; es decir, asociando la multimodalidad como sinónimo de b-learning (Turpo, 2010; Mortera, 2009) o de formación híbrida, lo cual no es considerado incorrecto aunque sí incompleto, pues la noción de formación multimodal en la UAEM, como se expresara, implica una gran amplitud de formatos o modalidades (100\% presenciales, 100\% virtuales y opciones híbridas).

En el $66 \%$ de las definiciones restantes, se aprecia una dispersión de concepciones entre las que se encuentran combinaciones completas de modalidades, que reflejan un mayor entendimiento del concepto, así como conceptualizaciones parciales, que equiparan la formación multimodal a la formación en ambientes virtuales, obviando incluir su componente presencial o que sólo focalizan los contenidos presenciales y virtuales, sin contemplar que la formación multimodal no sólo abarca contenidos, sino que necesariamente implica actividades de aprendizaje e interacciones entre los participantes. Asimismo, en este segmento se encontraron definiciones 
difusas y abstractas que revelan entendimiento limitado del concepto, evidente en expresiones tales como "diferentes alternativas de aprendizaje" o "proceso de innovación pedagógica".

Tradicionalmente, la formación abierta y a distancia han existido como modalidades alternativas a la escolarizada y por ello a los profesores les resulta distante y extraño imaginar la mezcla de todas las modalidades en una sola posibilidad, que es la formación multimodal, e insisten en considerar que ésta es una opción diferenciada de lo presencial e incluso, en ocasiones, ni siquiera la incluyen. Así también, desde un análisis más profundo, se observa en todas las definiciones, ausencia a la referencia de la dimensión filosófica y ética del concepto. Es decir, la noción de una formación centrada en el estudiante, flexible, inclusiva y enriquecida, mediante el aprovechamiento pedagógico de las ventajas de las diferentes modalidades educativas, con una visión innovadora, humanista, de compromiso social, de generación de saberes y abierta al mundo y a la diversidad.

Dicha información permitió visibilizar la necesidad de fortalecer, en el programa de formación docente para modalidades no convencionales de aprendizaje, las implicancias conceptuales en la práctica del concepto de formación multimodal para una efectiva implementación del mismo.

\subsection{Secretarios académicos de UA}

Otros de los actores que participaron en el proceso de la puesta en marcha de las asignaturas híbridas y virtuales en la UAEM fueron los secretarios académicos de las UA, por lo que se determinó necesario conocer cómo entendían el concepto de la Formación Multimodal ya que una de sus funciones es establecer la estrategia de la puesta en marcha de las UC en modalidades no convencionales. Para explorar sus conceptualizaciones la técnica que se utilizó fue la entrevista semi-estructurada, desde la cual se obtuvo la siguiente información:

El secretario académico Daniel ${ }^{4}$ definió el concepto formación multimodal en los siguientes términos: "es un idea muy buena" (entrevista personal, marzo 20, 2012).); el secretario académico Pablo al respecto dijo que es una "estrategia universitaria" y "espacio de formación" (entrevista personal, mayo 28, 2012); por último, la secretaria académica Martha lo definió como "sistema multimodal con posibilidad de incursionar en sistemas virtuales" y "cursos de forma presencial, virtual o cursos con parte presencial y parte virtual" (entrevista personal, marzo 3, 2012). Ésta última

\footnotetext{
4 Se utilizan nombres ficticios para dejar en el anonimato la identidad de los participantes.
} 
conceptualiza la definición de formación multimodal al enunciar que contempla cursos presenciales, virtuales y una combinación de ambos, sin embargo, obvia recuperar que, desde su génesis, e-UAEM no fue conceptualizado como un sistema independiente, sino como una vía de transformación de las formas tradicionales de enseñanza en la institución.

De la información que proporcionaron los entrevistados se infirió la ausencia de una compresión cabal del concepto de formación multimodal. Por tanto, es posible que ese desconocimiento fuera una línea de trabajo para analizar posibles causas de infrautilización en algunas UA y existencia de barreras en la puesta en marcha de asignaturas híbridas y virtuales, puestas de manifiesto en la aparición de conflictos en la operación de las mismas. Más adelante se abordan a detalle estas situaciones.

\subsection{Implementadores de e-UAEM}

Los implementadores son el equipo de trabajo de e-UAEM, conformado por la líder del proyecto, jefes de departamento, diseñadores formacionales, diseñadores multimedia y administradores de la plataforma. Se consideró necesario conocer cómo entendían el concepto del Espacio de la Formación Multimodal, ya que ellos tenían a su cargo su implementación y estaban en constante interacción con los directivos de la UAEM y docentes de las UA, por lo que se esperaba que tuvieran claro dicho concepto. Para explorar sus conceptualizaciones se utilizaron dos estrategias: la técnica de grupo focal, en el que participaron los diseñadores formacionales; asimismo se utilizó la entrevista semi-estructurada aplicada al resto del equipo de implementadores (líder del proyecto, coordinador académico, subcoordinador de producción y administrador de la plataforma), obteniendo lo siguiente:

Los diseñadores formacionales definieron la formación multimodal como: "distintas formas de aprender", "proceso de enseñanza-aprendizaje: presencial, virtual, híbrido o mixto" (grupo focal, noviembre 15, 2011). El diseñador formacional Luis lo definió como "oportunidad de transformación cultural" (entrevista personal, agosto 21, 2011) y el diseñador formacional David como "trayectoria que por momentos son presenciales, momentos híbridos y momentos virtuales" (entrevista personal, octubre 10, 2011).

A partir de lo presentado sobre el concepto de formación multimodal por parte de los sujetos que participaron en la investigación, se puede inferir que los actores con el rol de implementadores de e-UAEM son quienes manifestaron mayor claridad del concepto. 
Se puede concluir que, en el contexto del proyecto de la UAEM, la formación multimodal no es igual a educación a distancia, no es educación virtual, no es e-learning, no es b-learning, tampoco es m-learning, porque el concepto adoptado de forma explícita por la institución referido al tema de este trabajo (la multimodalidad) abarca, como ya se expresara, todas las modalidades desde lo $100 \%$ presencial hasta lo $100 \%$ virtual pasando por todo el intermedio (todo el intermedio sería b-learning). Zorrilla (2012: 4-5) lo define como "continuo de opciones de formación que van desde la oferta $100 \%$ presencial hasta la 100\% virtual, pasando por una variedad de combinaciones posibles entre éstas, todas consideradas formas híbridas o mixtas de aprendizaje." Ésta es la concepción que se sostiene respecto de la formación multimodal en el seno de e-UAEM, como estrategia institucional para ampliar la cobertura a partir de la oferta de programas educativos que combinan diferentes modalidades. De ahí la idea de considerarlo como una innovación ya que desafía las fronteras entre modalidades y plantea un escenario de flexibilidad modal que pretende aprovechar las ventajas que cada una de ellas representa.

Al tratarse de una innovación conceptual que difiere (aunque incluye) de las propuestas de educación a distancia, educación virtual, educación mixta, educación escolarizada y no escolarizada, para los actores de la UAEM ha resultado complejo interpretar y asimilar cómo en una propuesta "multimodal" es posible abarcar tres modalidades.

Al cierre de este trabajo la palabra "multimodal" ha permeado los discursos institucionales e incluso los ha trascendido, al ser incluida en una propuesta nacional presentada en 2013 por el Espacio Común de Educación Superior a Distancia (ECOESAD). No obstante, su significado sigue siendo trastocado por muchos que la utilizan como un término alternativo para referirse exclusivamente a la educación en línea y/o híbrida.

\section{CONCLUSIONES}

La implementación de un proyecto de innovación educativa a partir del concepto de multimodalidad fue compleja, ya que implicó el inicio de un proceso de cambio organizacional en las estructuras, procesos, cultura organizacional e infraestructura tecnológica. Uno de los primeros retos a los que se enfrentaron los implementadores de este proyecto fue el concepto de la formación multimodal, ya que los usuarios (directivos, administrativos, profesores y estudiantes) lo confundían con educación a distancia, educación abierta, educación híbrida, educación virtual, que si bien es 
cierto que se acercan a estos conceptos, no es lo mismo de acuerdo a lo que refiere Castillo (2014:102) "La formación multimodal no es igual a educación a distancia, no es educación virtual, no es e-learning, no es b-learning, no es m-learning, porque multimodal abarca todas las modalidades desde lo $100 \%$ presencial hasta lo $100 \%$ virtual pasando por todo el intermedio (todo el intermedio sería b-learning).

Se tuvo dificultad en dar a conocer a directivos, docentes y estudiantes un concepto con el que no estaban familiarizados. Los involucrados no lograban comprender en qué consistía la formación multimodal, lo cual trajo como consecuencia que a la hora de poner en marcha las asignaturas en modalidad virtual e híbrida, tuvieran diferentes situaciones para puesta en marcha de la innovación tecnológica. Ante esta situación fue necesario que e-UAEM continuara trabajando con las Unidades Académicas sobre el concepto de formación multimodal ya que aún existen conceptualizaciones erróneas o incompletas que ocasionan problemas a la hora de la operación de los programas educativos.

Se considera a la formación multimodal como una oportunidad de instalar un proceso de innovación institucional ya que desafía las fronteras entre modalidades y plantea un escenario de flexibilidad modal que pretende aprovechar las ventajas que cada una de ellas representa y podría ser una estrategia de expansión para las universidades Latinoamericanas considerando la necesidad de cobertura en el nivel Superior para la región. Por otra parte, con esta integración es posible flexibilizar y enriquecer la oferta presencial y cumplir con la meta de una educación inclusiva.

\section{BIBLIOGRAFÍA}

Acosta, R. C. (2002). Cuatro preguntas para iniciarse en cambio Organizacional. Revista Colombiana de Psicología, 11, 9-24.

Banco Mundial (2017). Noticia: La educación superior se expande en América Latina y el Caribe, pero aún no desarrolla todo su potencial. Mayo 17, 2017. Recuperado de: http://www.bancomundial.org/es/news/press-release/2017/05/17/highereducation-expanding-in-latin-america-and-the-caribbean-but-falling-short-ofpotential

Castillo (2014). Análisis del proceso de implementación de un entorno virtual de aprendizaje en educación superior: estudio de caso e-UAEM (Tesis de doctorado), México: Universidad Autónoma del Estado de Morelos.

ECOESAD (2013). Ejes para una política de educación media superior y superior a distancia con un enfoque multimodal. México: Espacio Común de Educación Superior a Distancia. 
Fernández, C. J. M. (2011). Multimodalidad y calidad educativa: Los retos de la construcción de conocimiento disciplinar en ambientes mediados por tecnología digital. Ponencia presentada en el XII Encuentro Internacional Virtual Educa, México, D. F.

García Aretio, L.(2002). Educación a distancia. De la teoría a la práctica. Barcelona: Ariel..

García Aretio, L. (2006). La Universidad Nacional de Educación a Distancia (UNED) de España (The National University of Distance Education (UNED) From Spain). Revista Iberoamericana de Educación a Distancia, 9(1/2), 17.

García, A. R. E. y Calderón, V. R. (2010). El Sistema de Educación Multimodal en la Universidad Veracruzana. Ponencia presentada en el X Congreso Nacional de Investigación Educativa (COMIE). Veracruz (2010)

Garrison (2007). Blended Learning in higher education. Agosto 13, 2007.

Kress, G. (2003) Literacy in the New Media Age. New York: Routledge.

Ley General de Educación (2006). Última Reforma DOF 28-01-2011. México. Consultado en: http://www.diputados.gob.mx/LeyesBiblio/pdf/137.pdf.

Mortera, G. F. J. (2009). El aprendizaje híbrido o combinado (Blended Learning): acompañamiento tecnológico en las aulas del siglo XXI. En Lozano R. A. y Burgos, A. J. V. (Eds.), Tecnología educativa en un modelo de educación a distancia centrado en la persona. México: Limusa.

Plan Nacional de Desarrollo 2007-2012 (2007). México. Consultado en: http://pnd.calderon. presidencia.gob.mx/pdf/PND 2007-2012.pdf

Turpo Gebera, O. W. (2010). Contexto y desarrollo de la modalidad educativa blended learning en el sistema universitario iberoamericano. Revista mexicana de investigación educativa, 15(45), 345-370

UAEM (2012). GACETA Año 17. Quinta época. núm. 420. 15 de marzo de 2012.

UNESCO-IESALC (2003). Informe Nacional sobre la Educación Superior en México. SEP: México. Consultado en: http://docenciasuperior.org/Documentos/Recursos/o publicaciones3.pdf

Zorrilla, A. M.L. (2009a). Universidad Flexible (febrero 2009). Universidad Autónoma del Estado de Morelos. Documento de trabajo.

Zorrilla, A. M.L. (2009b) Campus Virtual UAEM: Aprendizaje flexible para todos. 3er Congreso Internacional de Educación a Distancia Virtual "El e-learning al alcance de todos". Cuernavaca, Mor. 1 al 3 de abril de 2009.

Zorrilla, A. M.L. (2012). Conferencia: Formación Multimodal en la Universidad Autónoma del Estado de Morelos. En el tema contenidos y tecnologías de la información, la comunicación y el conocimiento por UNAM. 10/29/2012 - 20:08.

Zorrilla, A. M.L. y Castillo, D. M. (2013). Ponencia: Implementación de la formación multimodal en la UAEM: Primera etapa, en marco del XXI Encuentro Internacional de Educación a Distancia. Educación virtual en los cinco continentes. Realizada en la Universidad de Guadalajara del 2-6 de diciembre de 2013.

Zorrilla, A. M. L., García, P. O. y Castillo, D. M. (2013). Formación docente en línea a partir de una estrategia de producción de contenidos para ambientes virtuales de aprendizaje. Revista Apertura 5 (2). Consultado en: http://www.udgvirtual.udg. mx/apertura/index.php/apertura3/article/view/440/335. 